\title{
Importance of Polysynaptic Inputs and Horizontal Connectivity in the Generation of Tetanus-Induced Long-Term Potentiation in the Rat Auditory Cortex
}

\author{
Masaharu Kudoh and Katsuei Shibuki \\ Department of Neurophysiology, Brain Research Institute, Niigata University, Niigata 951, Japan
}

Supragranular pyramidal neurons in the adult rat auditory cortex (AC) show marked long-term potentiation (LTP) of population spikes after tetanic white matter stimulation (TS). For determination of whether this marked LTP is specific to AC, LTP in rat $A C$ slices was compared with LTP in slices of the visual cortex (VC). The amplitude of TS-induced LTP in AC was twice that in VC. LTP of EPSPs was also studied with perforated patch or whole-cell recording. Although the amplitude of TSinduced LTP of EPSPs in AC was larger that in VC, no cortical difference was found in LTP elicited by low-frequency stimulation paired with current injection. Neocortical LTP is dependent on the activation of NMDA receptors, and induction of LTP requires postsynaptic depolarization for removal of $\mathrm{Mg}^{2+}$ blockade of NMDA receptors. The postsynaptic depolarization elicited by TS in supragranular pyramidal neurons in $A C$ was significantly larger than that in VC. Cutting of supragranular horizontal connections resulted in a decrease in the depolarization amplitude in $\mathrm{AC}$ but an increase in the depolarization amplitude in VC. The cortical difference in TS-induced LTP was diminished in the slices in which horizontal connections in supragranular layers were cut. The estimated density of horizontal axon collaterals of supragranular pyramidal neurons in AC was approximately twice that in VC. These results strongly suggest that the marked polysynaptic and postsynaptic depolarization during TS and the resulting marked LTP in AC are attributed to well developed horizontal axon collaterals of supragranular pyramidal neurons in AC.

Key words: auditory cortex; long-term potentiation; pyramidal neuron; axon collateral; horizontal connection; visual cortex
Experience-dependent synaptic plasticity is required for development of the primary sensory cortex (Hubel and Wiesel, 1963; Wiesel and Hubel 1963; Blakemore and Cooper, 1970; Hirsch and Spinelli 1970; Woolsey and Wann, 1976). Long-term potentiation (LTP) of thalamocortical synapses is elicited in young animals (Crair and Malenka, 1995). Cortical LTP in young animals is thought to serve as a cellular mechanism for developmental plasticity (Kirkwood et al., 1995). LTP of intracortical circuits is elicited in adult animals (Iriki et al., 1989; Hirsch and Gilbert, 1993; Hess and Donoghue, 1994). Neocortical LTP is usually dependent on the activation of NMDA receptors (Artola and Singer, 1987; Kimura et al., 1989), and postsynaptic depolarization is required for the induction of LTP to remove $\mathrm{Mg}^{2+}$ blockade of NMDA receptors (Norwak et al., 1984; Artola et al., 1990). Postsynaptic depolarization is also required for inducing the plasticity of neural responsiveness in visual cortex (VC) (Frégnac et al., 1992; Shultz and Frégnac, 1992) and in auditory cortex (AC) (Cruikshank and Weinberger, 1996).

It has been reported that the extents of neocortical LTP and postsynaptic depolarization during induction of LTP are different between the motor and somatosensory cortices (CastroAlamancos et al., 1995; Castro-Alamancos and Connors, 1996).

Received Aug. 8, 1997; revised Sept. 29, 1997; accepted Sept. 30, 1997.

This work was supported by grants from the Japanese Government, Toyota RIKEN, the Uehara Foundation, and the Nissan Science Foundation. We thank T. Bando and $\mathrm{H}$. Nawa for reading this manuscript and $\mathrm{Y}$. Tamura and N. Taga for technical assistance.

Correspondence should be addressed to Katsuei Shibuki, Department of Neurophysiology, Brain Research Institute, Niigata University, 1 Asahi-machi, Niigata 951, Japan.

Copyright (C) 1997 Society for Neuroscience $\quad 0270-6474 / 97 / 179458-08 \$ 05.00 / 0$
Therefore, it is quite likely that functional characteristics of each cortex are reflected in the diversity of synaptic plasticity. In AC of adult animals, the receptive field properties of single neurons change on sound discrimination learning (e.g., Edeline and Weinberger, 1993), and the cortical region responding to tones of the frequency used enlarges (Recanzone et al., 1993). Synaptic connections between auditory cortical neurons are potentiated by correlated activities during learning (Ahissar et al., 1992). A short auditory experience elicits synaptic plasticity in AC, by which the sound discrimination ability of rats is enhanced (Sakai et al., 1995, 1996). Marked LTP of population spikes is observed in AC of adult rats (Kudoh and Shibuki, 1994, 1996a). These many findings raise the possibility that $\mathrm{AC}$ of adult animals may be specialized to produce marked synaptic plasticity.

Pyramidal neurons are connected to each other via axon collaterals (Thomson and Deuchars, 1994), and thalamocortical inputs are amplified by excitatory recurrent circuits (Douglas et al., 1995). During presentation of a species-specific vocalization, synchronized neural activities are recorded from a wide area of AC of the common marmoset (Wang et al., 1995). Correlated activities between a pair of neurons are detected in AC during presentation of auditory stimuli (deCharms and Merzenich, 1996). Well developed connections between pyramidal neurons, the presence of which in AC are expected based on these findings, may play a critical role in amplification of postsynaptic depolarization and induction of synaptic plasticity. Therefore, we compared LTP between AC and VC and studied the relationship between the diversity of LTP and that of intracortical circuits.

Preliminary results have been published in abstract form (Kudoh and Shibuki, 1996b). 


\section{MATERIALS AND METHODS}

Materials. Wistar rats of both sexes (4-7 weeks old) were used. After a rat was anesthetized with ether, it was immersed in ice-cold water except for the nose for $3 \mathrm{~min}$ to reduce brain temperature. Immediately after decapitation, the brain was removed in an ice-cold artificial medium bubbled with $95 \% \mathrm{O}_{2}$ and $5 \% \mathrm{CO}_{2}$. The composition of the medium (in $\mathrm{mm}$ ) was $\mathrm{NaCl} 124, \mathrm{KCl} 5, \mathrm{NaH}_{2} \mathrm{PO}_{4} 1.24, \mathrm{MgSO}_{4} 1.3, \mathrm{CaCl}_{2} 2.4$, $\mathrm{NaHCO}_{3} 26$, and glucose 10. AC was determined as area 41 (Krieg, 1964) or area 1 of the temporal cortex (Zilles, 1985), and a block including AC was dissected out (see Fig. $1 A$ ). Coronal slices (thickness, $400 \mu \mathrm{m}$ ) were prepared from the block with a microslicer (DTK-2000; Dosaka, Osaka, Japan). For comparison, coronal slices were prepared from VC (see Fig. $1 A$ ), which was identified as area 17 (Krieg, 1964) or area 1 of the occipital cortex (Zilles, 1985). In some experiments, slices cut in a plane orthogonal to the coronal plane and the surface of the cortex were prepared from $\mathrm{AC}$ and $\mathrm{VC}$, respectively. The obtained slices were incubated in an artificial medium at $30^{\circ} \mathrm{C}$ for at least $1 \mathrm{hr}$ before recording. The recording was performed at $35^{\circ} \mathrm{C}$ unless otherwise specified. During the recording, the slices were continuously perfused with the oxygenated medium at a flow rate of $1 \mathrm{ml} / \mathrm{min}$. In some experiments, various drugs were added to the perfusing medium. 6-Cyano-7-nitroquino xaline-2,3-dione (CNQX) and D-(-)-2-amino-5-phosphonovalerate (DAPV) were obtained from Tocris Cookson (Bristol, UK). Bicuculline was purchased from Research Biochemicals (Natick, MA). The experiments were performed according to the guidelines of Niigata University and had the approval of the ethics committee of Niigata University.

Stimulation. As a stimulating electrode, the cut end of a Teflon-coated Pt wire (metal diameter, $50 \mu \mathrm{m}$ ) was placed on the border between the white matter and layer VI. An electrolytically polished Ag wire, which was insulated with polyvinyl chloride except for the tip, was also used as a stimulating electrode and inserted into the border between the white matter and layer VI. In some experiments, supragranular layers were stimulated with these electrodes. Biphasic pulses (duration of each phase of each pulse, $100 \mu \mathrm{sec}$ ) were passed through the electrode to minimize stimulus artifacts in the recording. The intensity of pulses in each phase was $600 \mu \mathrm{A}$ for field potential recording unless otherwise specified. For whole-cell or perforated patch recording, the intensity of the stimuli was $150 \mu \mathrm{A}-1 \mathrm{~mA}$. For evoking LTP in field potentials, tetanic white matter stimulation (TS; 100 pulses at $100 \mathrm{~Hz}$, twice at a $30 \mathrm{sec}$ interval) or $\theta$-burst stimulation (four pulses at $100 \mathrm{~Hz}$, five pulse trains at $5 \mathrm{~Hz}$, repeated five times at $0.1 \mathrm{~Hz}$ ) was applied to the white matter. For evoking LTP in EPSPs of pyramidal neurons, TS (100 pulses at $100 \mathrm{~Hz}$, $500 \mu \mathrm{A}$ ) was used. Low-frequency white matter stimulation (100 pulses at $2 \mathrm{~Hz}, 150 \mu \mathrm{A})$ paired with injection of depolarizing current $(1.5 \mathrm{nA}, 100$ msec) was also used to elicit LTP in EPSPs. In this experiment, a stimulus pulse was applied to the white matter $50 \mathrm{msec}$ after initiation of the current injection for $100 \mathrm{msec}$.

Field potential recording. Field potentials elicited by white matter stimulation or supragranular focal stimulation were recorded through an electrolytically polished $\mathrm{Ag}$ wire, which was insulated with polyvinyl chloride except for the tip. After being passed through a bandpass filter between $0.2 \mathrm{~Hz}$ and $10 \mathrm{kHz}$, the data were stored in a computer for later analysis. In some experiments performed using bicuculline $(0.5 \mu \mathrm{M})$, the stimulus intensity was reduced to 300-400 $\mu \mathrm{A}$ for adjustment of the amplitude of field potentials, and additional currents were applied during TS through another stimulating electrode close to the other to give a total current of $600 \mu \mathrm{A}$.

Whole-cell or perforated patch recording. Supragranular pyramidal neurons were recorded using a blind slice patch technique similar to the one described previously (Kudoh and Shibuki, 1996a). A glass micropipette was filled with a solution containing (in mM): K-gluconate 130, KCl 10, HEPES 10, EGTA 1 (or 0.5 for recording LTP), $\mathrm{MgCl}_{2} 1$, Na-ATP 4 Na-GTP 1 , and sucrose 16 , with $\mathrm{pH}$ adjusted to 7.2 with $\mathrm{KOH}$ (resistance, 20-30 M $\Omega$ ). This electrode was inserted into supragranular layers, and a positive pressure of $\sim 30 \mathrm{mmHg}$ was applied in the pipette during insertion. After a sudden increase in the access resistance of the electrode, negative pressure was applied to obtain whole-cell current-clamp recording.

For recording LTP in a pyramidal neuron, perforated patch recording was also used. The composition of the filling medium was (in mM): $\mathrm{K}_{2} \mathrm{SO}_{4}$ 92, $\mathrm{KCl} 31$, HEPES 10 , and $\mathrm{MgCl}_{2} 1$, adjusted to $\mathrm{pH} 7.4$ with $\mathrm{KOH}$. The resistance of the recording electrode was 8-12 $\mathrm{M} \Omega$. Amphotericin B (Wako Chemicals) dissolved in dimethylsulfoxide $(10 \mathrm{mg} / \mathrm{ml})$ was added to the patch medium (final concentration, $50 \mu \mathrm{g} / \mathrm{ml}$ ). The tip was filled with an amphotericin B-free medium to facilitate the sealing between the pipette and cell membrane. Membrane resistance and resting membrane potential were measured, and neurons with a resting membrane potential more negative than $-55 \mathrm{mV}$ were selected. Pyramidal neurons were identified in terms of an antidromic spike elicited by white matter stimulation at an intensity $\leq 1 \mathrm{~mA}$ (Kudoh and Shibuki, 1996a). The recording was performed at $30^{\circ} \mathrm{C}$ for LTP experiments and at $35^{\circ} \mathrm{C}$ for other experiments.

\section{RESULTS}

\section{Comparison of synaptic responses elicited by single-pulse stimulation}

Before study of the diversity of LTP, synaptic responses elicited by single-pulse stimulation were compared between AC and VC. The field potentials elicited by white matter stimulation in supragranular layers of $\mathrm{AC}$ are composed of early and late negative waves (Fig. $1 B$ ). The early negativity was resistant to $10 \mu \mathrm{M}$ CNQX, an antagonist of non-NMDA glutamate receptors, whereas the late negativity was blocked by CNQX as described previously (Kudoh and Shibuki, 1994). This CNQX-sensitive negativity corresponds to trans-synaptically activated population spikes of pyramidal neurons (Kudoh and Shibuki, 1996a). These two components were recorded in both cortices (Fig. 1B,C). The amplitude of the CNQX-resistant or CNQX-sensitive component was separately measured in $\mathrm{AC}(2.08 \pm 0.17$ and $0.85 \pm 0.08 \mathrm{mV}$, mean $\pm \mathrm{SEM} ; n=15)$ and in $\mathrm{VC}(2.07 \pm 0.19$ and $0.92 \pm 0.09 \mathrm{mV}$; $n=11)$. However, no significant difference between AC and VC was found regarding the amplitude of each component. The third component appeared in an $\mathrm{Mg}^{2+}$-free medium containing $10 \mu \mathrm{M}$ CNQX (Fig. 1B,C). The third component was blocked by $50 \mu \mathrm{M}$ D-APV, an antagonist of NMDA receptors. The amplitude of this D-APV-sensitive component in AC $(0.63 \pm 0.11 \mathrm{mV} ; n=5)$ was not significantly different from that in $\mathrm{VC}(0.68 \pm 0.16 \mathrm{mV} ; n=5)$.

The difference in synaptic responses between the cortices was analyzed using whole-cell current-clamp recording. The resting membrane potential was $-61 \pm 1 \mathrm{mV}(n=25)$, and the membrane resistance was $45 \pm 3 \mathrm{M} \Omega$ in the supragranular pyramidal neurons in $\mathrm{AC}$, whereas these parameters were $-61 \pm 1 \mathrm{mV}(n=$ 26) and $43 \pm 2 \mathrm{M} \Omega$, respectively, in VC. For comparison of the amplitudes of EPSPs that did not accompany antidromic spikes, white matter was weakly stimulated at an intensity of $150 \mu \mathrm{A}$. The rate of rise of EPSP amplitude was $1.8 \pm 0.2 \mathrm{~V} / \mathrm{sec}(n=17)$ in $\mathrm{AC}$ and $2.1 \pm 0.2 \mathrm{~V} / \mathrm{sec}(n=16)$ in $\mathrm{VC}$, and no significant difference between the cortices was found regarding these data.

\section{Comparison of LTP between the cortices}

LTP was compared in coronal slices of AC and VC. Only when the CNQX-resistant and trans-synaptic components of the field potentials elicited by the white matter stimulation at the intensity of $600 \mu \mathrm{A}$ were $>1.5$ and $0.5 \mathrm{mV}$, respectively, was analysis of LTP performed. As reported earlier (Kudoh and Shibuki, 1994), LTP in AC was selectively elicited by TS in the trans-synaptic population spikes, and the CNQX-resistant field potentials did not change on induction of LTP (Fig. 1D). LTP was observed in the slices obtained from rats of widely distributed ages (29-49 d postnatal), and no particular critical period was found in this range of ages. Similarly, LTP was recorded in VC, but the amplitude of this LTP was smaller than that of the LTP in AC (Fig. $1 E$ ). The LTP amplitude was estimated $30 \mathrm{~min}$ after cessation of TS. The value was $51 \pm 8 \%(n=15)$ in AC and $26 \pm 5 \%(n=$ 11) in VC (Fig. $1 F, G)$, and the difference was statistically significant (Mann-Whitney $U$ test, $p<0.01$ ).

The observed diversity in TS-induced LTP might reflect a trivial difference in the recording conditions rather than a fun- 
A

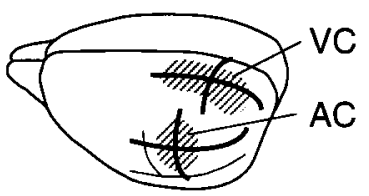

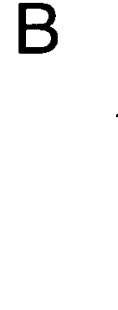

Figure 1. Supragranular field potentials elicited by singlepulse stimulation and TS-induced LTP in AC and VC. $A$, Diagram showing AC and VC. Solid lines in AC and VC represent the planes from which slices were prepared. $B$, Field potentials in AC. Three traces are superimposed, which were recorded in normal medium, in $\mathrm{Mg}^{2+}$-free medium containing $10 \mu \mathrm{M} \mathrm{CNQX}$, and in $\mathrm{Mg}^{2+}$-free medium containing CNQX and $50 \mu \mathrm{M}$ D-APV. $C$, Field potentials in VC. The three traces were recorded under the same conditions as in $B$. $D$, Field potential traces in AC recorded before and after $\left(^{*}\right)$ TS. Note the potentiation evoked in the trans-synaptic component of field potentials selectively. Two traces elicited every $30 \mathrm{sec}$ were averaged to estimate the field potentials. $E$, Field potentials in VC recorded before and after $(*)$ TS. F, Time course of LTP in AC (filled circles) and VC (open circles). Mean and SEM are shown. Numbers of the experiments are shown in parentheses. $G$, Amplitude of LTP in AC (hatched bars) and VC (open bars) elicited by TS in coronal slices $(T S)$ or in the slices orthogonal to the coronal plane $\left(T S^{*}\right)$. LTP elicited in the coronal slices by $\theta$-burst stimulation ( $\theta$-burst $)$ or by TS in the presence of bicuculline $(0.5 \mu \mathrm{M} ; B I C)$ is shown. Asterisks represent significant differences $(p<0.01$ or 0.05 ) between $\mathrm{AC}$ and VC.

Figure 2. LTP in the rising slope of EPSPs recorded in pyramidal neurons. $A$, EPSPs recorded before and after $(*)$ TS in AC. The intensity of the test stimuli was $150 \mu \mathrm{A}$. $B$, EPSPs recorded before and after (*) TS in VC. $C$, Time course of TS-induced LTP in AC (filled circles) and in VC (open circles). Mean and SEM are shown. $D$, EPSPs recorded before and after $(*)$ low-frequency stimulation paired with depolarizing current injection in AC. E, EPSPs before and after $(*)$ low-frequency stimulation plus depolarizing current injection in VC. F, Time course of LTP elicited by a combination of low-frequency stimulation and current injection in $\mathrm{AC}$ ( filled circles) and in VC (open circles). In $A-C$, pyramidal neurons were recorded using a perforated patch technique. In $D-F$, a conventional whole-cell recording method was used.
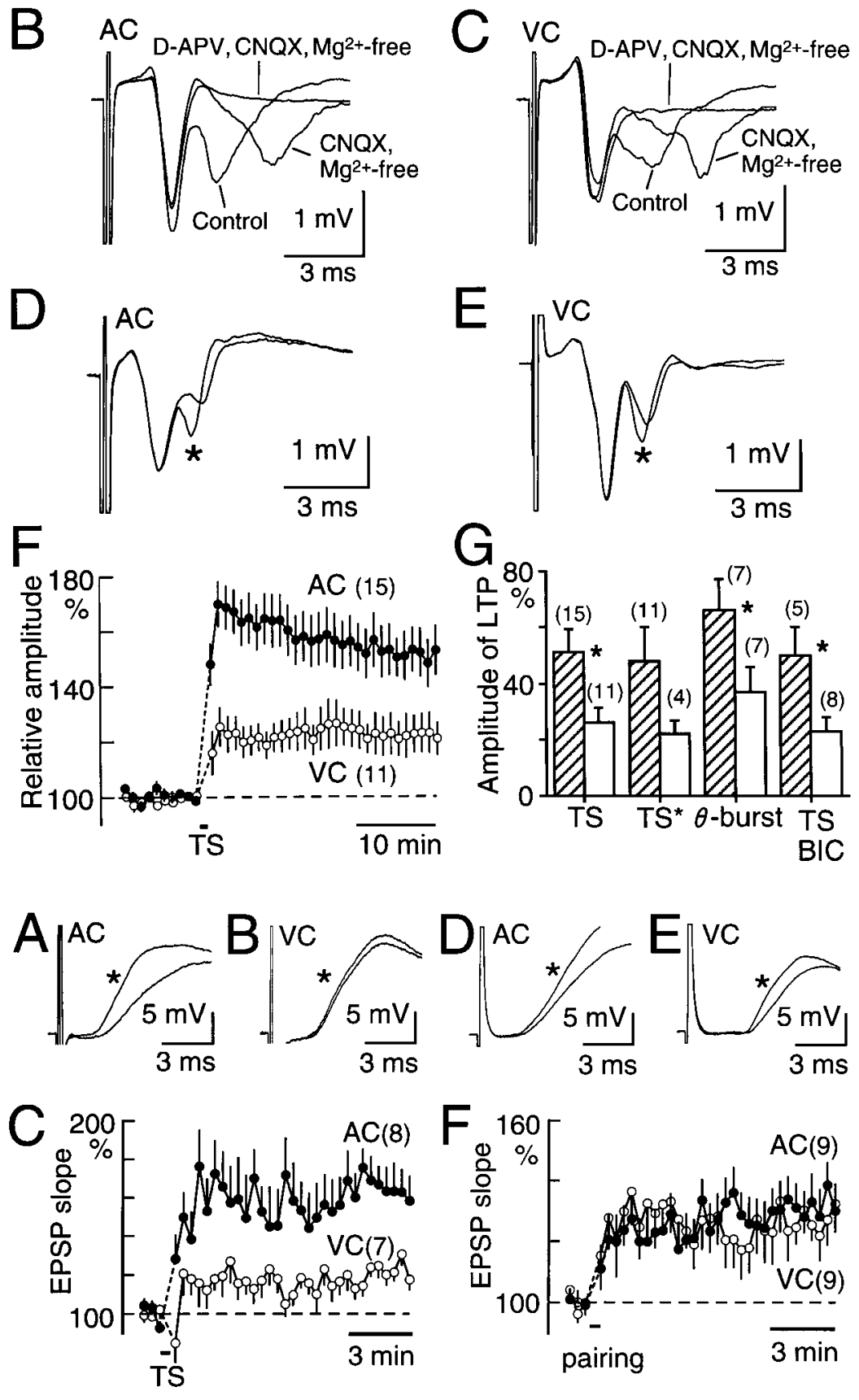

damental difference between the cortices. For investigation of this possibility, LTP recorded under various conditions was compared. First, LTP in AC and VC was recorded in horizontal and parasagittal slices, respectively. However, the amplitude of the LTP was not significantly different from that of the LTP recorded in coronal slices (Fig. 1G). The amplitude of LTP in horizontal slices of AC was twice that of the LTP in parasagittal slices of VC (Fig. 1G), although the difference in LTP amplitude between AC and VC was not statistically significant in these slices. In coronal slices, LTP was also elicited by $\theta$-burst stimulation or recorded in the presence of bicuculline $(0.5 \mu \mathrm{M})$, an antagonist of $\mathrm{GABA}_{\mathrm{A}}$ receptors, and a significant difference in LTP amplitude between AC and VC was observed in both experiments ( $p<0.05$; Fig. $1 G$ ).

We compared LTP in the rising slope of EPSPs with perforated patch recording from pyramidal neurons (Fig. $2 A-C$ ). The LTP amplitude was estimated $10 \mathrm{~min}$ after cessation of TS, because recording conditions in many neurons were stable within this period. Again, there was a significant difference $(p<0.05)$ in 

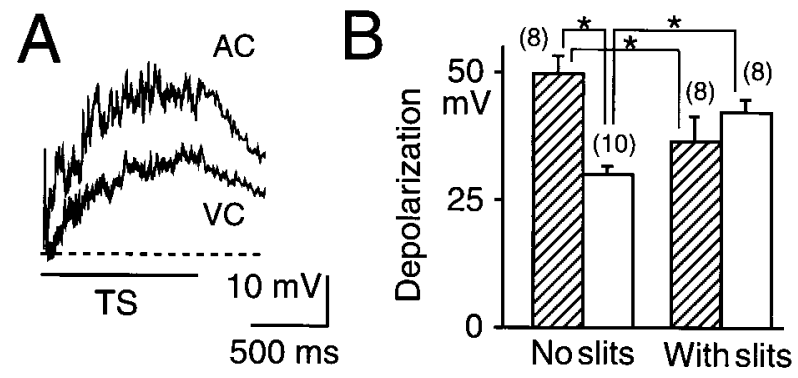

Figure 3. Depolarization of supragranular pyramidal neurons in response to TS (100 pulses at $100 \mathrm{~Hz}, 600 \mu \mathrm{A})$. $A$, Typical traces recorded in $\mathrm{AC}$ and $\mathrm{VC}$ are superimposed. The broken line shows the resting membrane potential. $B$, Amplitudes of depolarization induced by TS in $\mathrm{AC}$ (hatched bars) and VC (open bars). The amplitudes of depolarization recorded in the slices in which the horizontal connections in supragranular layers were interrupted by a pair of slits $450 \mu \mathrm{m}$ apart are also shown. Pairs of data in which a significant difference ( $p<0.01$ or 0.05 , MannWhitney $U$ test) was found are marked by asterisks.

TS-induced LTP amplitude between AC $(61 \pm 10 \% ; n=8)$ and $\mathrm{VC}(23 \pm 4 \% ; n=7)$. These results clearly indicate that there is a significant cortical difference in TS-induced LTP amplitude, regardless of the method used for recording LTP. However, when LTP was elicited by low-frequency white matter stimulation paired with injection of depolarizing current (Fig. $2 D-F)$, there was no apparent difference in the LTP amplitude between AC $(32 \pm 8 \% ; n=9)$ and VC $(29 \pm 8 \% ; n=9)$. Although no significant difference between the cortices was found regarding the membrane resistance of pyramidal neurons, these results suggest a possibility that the cortical difference in TS-induced LTP may be attributed to that in the amplitude of depolarization of pyramidal neurons during TS.

\section{Diversity of polysynaptic and postsynaptic depolarization and roles of horizontal connections in the diversity}

The diversity in TS-induced LTP between AC and VC suggests the presence of a difference in the extent of postsynaptic depolarization during TS. Therefore, we recorded supragranular pyramidal neurons with whole-cell recording and measured the postsynaptic depolarization during TS (Fig. $3 A$ ). The maximal amplitude of the depolarization during TS was $50 \pm 3 \mathrm{mV}(n=$ $8)$ in $\mathrm{AC}$, whereas it was $30 \pm 1 \mathrm{mV}(n=10)$ in $\mathrm{VC}$, and a significant difference was found between the two values $(p<$ 0.01; Fig. 3B).

Because no significant difference between the cortices was found in the synaptic responses elicited by single-pulse stimulation or in the membrane resistance of pyramidal neurons, we expected that the diversity in the depolarization was attributable to polysynaptic excitation (Castro-Alamancos et al., 1995), which may be amplified in intrinsic neural circuits (Douglas et al., 1995). Possible pathways responsible for the diversity in TS-induced LTP are recurrent excitatory connections between pyramidal neurons through horizontal axon collaterals. Therefore, horizontal connections were cut by making a pair of slits $450 \mu \mathrm{m}$ apart in the supragranular layers of the slices. The slits were made using a razor blade attached to a manipulator. The amplitude of the depolarization in $\mathrm{AC}$ was significantly reduced by the presence of the slits $(p<0.05$; Fig. $3 B)$, whereas that in $\mathrm{VC}$ was significantly augmented $(p<0.05)$. These data suggest that the differences in the extent of polysynaptic and postsynaptic depolarization may be derived from synaptic inputs mediated by horizontal connections.

\section{Roles of horizontal connections in diversity of TS-induced LTP}

Because the difference in TS-induced depolarization of pyramidal neurons was diminished by the presence of a pair of slits, we cut supragranular horizontal connections by adding a pair of slits and measured the amplitude of TS-induced LTP. The LTP amplitude in AC was significantly reduced by the presence of a pair of slits 400 or $450 \mu \mathrm{m}$ apart (Fig. $4 A$ ). However, no effect of adding slits was observed in VC (Fig. 4B). The size of the gap between the slits was varied. The amplitude of LTP in AC was significantly reduced by the presence of a pair of slits 400 or 450 $\mu \mathrm{m}$ apart ( $p<0.05$, respectively) but not $500 \mu \mathrm{m}$ apart (Fig. $4 C$ ). The LTP in VC was not affected significantly by the presence of a pair of slits with any of the interslit gap sizes used, and therefore, no significant difference in LTP amplitude between AC and VC was observed in the slices with a pair of slits 400 or 450 $\mu \mathrm{m}$ apart. These data strongly support the hypothesis that horizontal connections play a facilitative role in induction of TSinduced LTP in AC but not in VC.

\section{Horizontal spreading of trans-synaptic field potentials in supragranular layers}

For study of synaptic responses mediated by horizontal connections in supragranular layers, recording and stimulating electrodes were placed in supragranular layers, and horizontal spreading of the field potentials in response to focal stimulation was recorded. CNQX-resistant and trans-synaptic components of the field potentials were observed after the focal stimulation at an intensity of $300 \mu \mathrm{A}$ in both cortices (Fig. 5A). The distance between the stimulating and recording electrodes was varied, and the relationship between the distance and the amplitude of the trans-synaptic field potentials was plotted (Fig. $5 A$ ). The potentials in $\mathrm{AC}$ were larger than those in $\mathrm{VC}$, and a significant difference in the amplitude $(p<0.05)$ was found at 400,500 , and $600 \mu \mathrm{m}$ (Fig. 5A). For determination of the contribution of intrinsic inhibition to the difference, field potentials were recorded in the presence of bicuculline $(0.5 \mu \mathrm{M})$. Horizontal spreading of the trans-synaptic potentials was facilitated by bicuculline (Fig. 5B). Although the effect of bicuculline was observed in both cortices, it was more pronounced in VC than in AC, and no significant difference in the amplitude of trans-synaptic responses between the cortices was observed in the presence of bicuculline (Fig. $5 B$ ).

\section{Horizontal spreading of CNQX-resistant potentials in supragranular layers}

Although no clear difference in the trans-synaptic field potentials was found between $\mathrm{AC}$ and $\mathrm{VC}$ in the presence of bicuculline (Fig. 5B), a significant difference in TS-induced LTP amplitude between $\mathrm{AC}$ and $\mathrm{VC}$ was observed even in the presence of bicuculline (Fig. 1G). Therefore, we focused on horizontal spreading of CNQX-resistant field potentials. We recorded the amplitude of the field potentials in the presence of CNQX (10 $\mu \mathrm{M}$; Fig. $5 C, D$ ). For evoking clear potentials, focal stimulation at an intensity of $600 \mu \mathrm{A}$ was used. The amplitude of the CNQXresistant potentials in $\mathrm{AC}$ was larger than that in VC. It was $2.1 \pm$ $0.4 \mathrm{mV}(n=7)$ in $\mathrm{AC}$ and $1.3 \pm 0.2 \mathrm{mV}(n=9)$ in VC at a site $300 \mu \mathrm{m}$ from the stimulating electrode, and the difference was significant $(p<0.05$; Fig. $5 C, D)$. The CNQX-resistant field potentials are composed of presynaptic volley potentials and antidromic population spikes. Therefore, it is suggested that the density of horizontal connections is higher in AC than in VC. 
Figure 4. Effects of the presence of a pair of slits made in the supragranular layers on TS-induced LTP. $A$, Time course of LTP in AC recorded in the slices with or without a pair of slits 400 or $450 \mu \mathrm{m}$ apart (filled circles). TS was used to evoke LTP. Control LTP is also shown (open circles). B, Time course of LTP recorded in VC with ( filled circles) or without (open circles) a pair of slits. $C$, The relationship between the interslit gap size $(400-500 \mu \mathrm{m})$ and the amplitude of LTP. Filled and open circles represent data recorded in AC and VC, respectively. Asterisks mark pairs of data in which a significant difference was found $(p<0.05)$.
Figure 5. Horizontal spreading of trans-synaptic field potentials recorded in the supragranular layers after focal stimulation at $300 \mu \mathrm{A}$. A, Relationship between the amplitude of the trans-synaptic field potentials and the distance between the recording and stimulating electrodes in $\mathrm{AC}$ (closed circles) and VC (open circles). Mean and SEM are shown. Asterisks represent significant differences between $\mathrm{AC}$ and $\mathrm{VC}(p<$ $0.05)$. Insets, Traces of field potentials recorded in $\mathrm{AC}$ and VC. Arrowheads represent the trans-synaptic field potentials. The distance between the recording and stimulating electrodes was $300 \mu \mathrm{m}$. $B$, Data similar to those in $A$, recorded in the presence of bicuculline $(0.5 \mu \mathrm{M})$. $C$, Horizontal spreading of supragranular CNQX-resistant field potentials elicited by focal stimulation at $600 \mu \mathrm{A}$ in AC. Relationship between the amplitude of CNQX-resistant potentials and the distance between electrodes is plotted. Data recorded in the same slice are represented by the same symbols connected by lines. Inset, Trace of field potentials recorded in the supragranular layers of AC. For blocking trans-synaptic potentials and subsequent activation of inhibitory neurons, recording was performed in the presence of $10 \mu \mathrm{M}$ CNQX. $D$, Data similar to those in $C$, recorded in $\mathrm{VC}$.

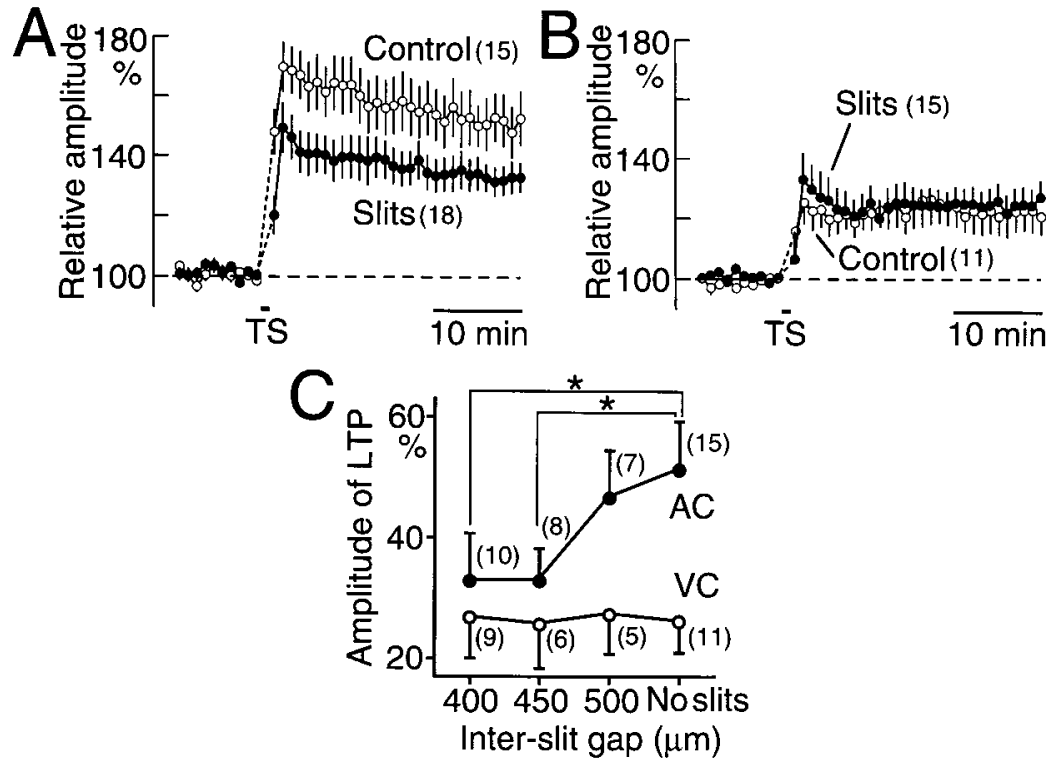

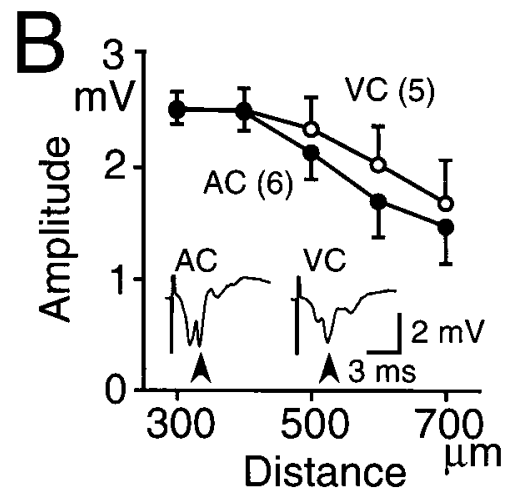

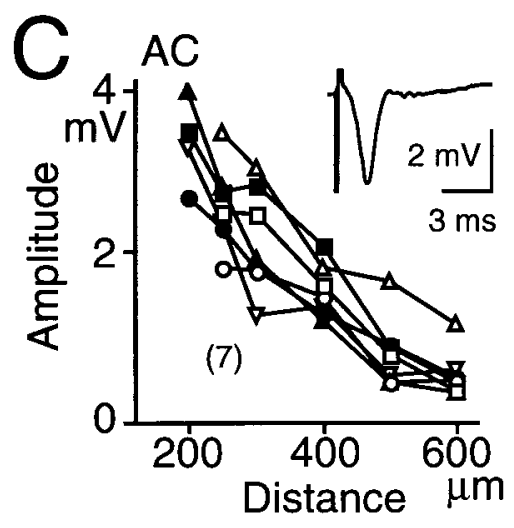

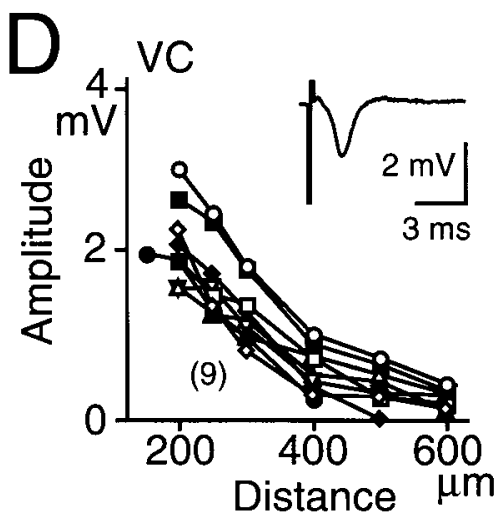

For confirmation of the difference in the density of horizontal connections, whole-cell recording was used. Focal stimulation of supragranular layers produced EPSPs in a typical supragranular pyramidal neuron in each cortex (Fig. 6). As the stimulus intensity was increased, the amplitude of the EPSPs increased, and orthodromic spikes appeared. In some neurons, an antidromic spike appeared without a preceding EPSP (Fig. 6A). Of 35 supragranular pyramidal neurons recorded in AC, 18 showed antidromic spiking in response to the $600 \mu \mathrm{A}$ focal stimulation, whereas only 6 of 25 visual neurons responded antidromically. This difference in the ratio of neurons showing antidromic spiking between the cortices was significant $\left(\chi^{2}\right.$ test, $\left.p<0.05\right)$. It is concluded from these results that the density of horizontal axon collaterals of pyramidal neurons in $\mathrm{AC}$ is approximately twice that in $\mathrm{VC}$.

\section{DISCUSSION}

In our previous study (Kudoh and Shibuki, 1996a), we found that most of supragranular pyramidal neurons recorded in AC slices of adult rats showed marked TS-induced LTP in trans-synaptic spiking activities, which correspond to cortical output to other cortical areas (Toyama et al., 1969). For determination of whether this marked TS-induced LTP is specific to AC, we compared LTP between $\mathrm{AC}$ and $\mathrm{VC}$ in the present study and found that TSinduced LTP in AC was twice that in VC, whereas no cortical 


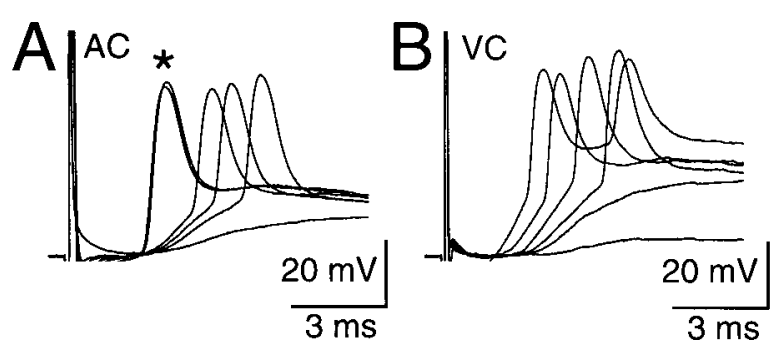

Figure 6. Responses of supragranular pyramidal neurons elicited by focal stimulation of the supragranular layer $300 \mu \mathrm{m}$ from the recording electrode. $A$, Traces recorded in AC. The stimulus intensity was between 120 and $320 \mu \mathrm{A}$. The asterisk represents antidromic spikes. $B$, Data recorded in $\mathrm{VC}$ elicited by stimuli with an intensity of $150-800 \mu \mathrm{A}$.

difference was found in LTP of EPSPs elicited by low-frequency stimulation paired with current injection. Results of analyses for the difference in TS-induced LTP amplitude led us to conclude that polysynaptic and postsynaptic depolarization in pyramidal neurons of AC is amplified by activity of intrinsic local circuits composed of well developed horizontal axon collaterals, and that marked TS-induced LTP in AC is attributable to the amplified postsynaptic depolarization during TS. The rationale for this conclusion and possible functions of well developed horizontal connections in AC are discussed below.

\section{Linkage between TS-induced postsynaptic depolarization and TS-induced LTP}

We tested various possibilities that might explain the cortical difference in TS-induced LTP amplitude. The possible technical artifacts generated during preparation of the slices might produce the difference. However, TS-induced LTP amplitude recorded in horizontal slices of AC and parasagittal slices of VC did not differ markedly from those recorded in coronal slices. It is also unlikely that synaptic responses elicited by single-pulse stimulation differ significantly between the cortices. Although NMDA receptors are quite important for evoking neocortical LTP in AC (Kudoh and Shibuki, 1994, 1996a) and VC (Artola and Singer, 1987; Kimura et al., 1989), no clear difference in the amplitude of the population spikes elicited by activation of NMDA receptors was found between the cortices. Diversity in intrinsic inhibition is another possibility, but the difference in TS-induced LTP was not diminished by bicuculline (Fig. $1 G$ ). However, no apparent cortical difference was found in pairing-induced LTP of EPSPs (Fig. $2 F$ ). This finding strongly supports the notion that the difference in TS-induced LTP is a result of circuitry rather than biochemical differences. Similarly, the difference in TS-induced LTP cannot be explained by cortical diversities in the critical period for the induction of LTP. The remaining possibility is that the difference in TS-induced LTP is derived from differences in the extents of TS-induced postsynaptic depolarization. In the slices in which supragranular horizontal connections were interrupted by a pair of slits, the difference in TS-induced LTP was diminished, and TS-induced depolarization was reduced in AC and augmented in VC. A causal linkage between the TS-induced postsynaptic depolarization and TS-induced LTP is strongly suggested by these results.

\section{Roles of horizontal axon collaterals of pyramidal neurons in generation of LTP diversity}

TS-induced depolarization was reduced by the presence of slits in AC but was augmented in VC. Therefore, it is expected that more inhibitory neurons are activated by horizontally propagating in- puts in VC than in AC. This hypothesis is consistent with the observation that the supragranular field potentials elicited by focal stimulation were augmented by bicuculline, as shown previously (Chagnac-Amitai and Connors, 1989), and that the effect of bicuculline is more pronounced in VC than in AC (Fig. 5A,B). However, LTP in VC was not augmented by bicuculline or by cutting of horizontal connections in the supragranular layers in the present study. This apparent discrepancy may be explained by the presence of inhibitory inputs terminating on the soma and the initial segment of pyramidal neurons (Williams et al., 1992). These inhibitory inputs are expected to suppress spike generation regardless of excitatory dendritic inputs, whereas dendritic depolarization and resulting LTP at excitatory synapses on remote dendrites are not (Blomfield, 1974). The density of horizontal connections can be estimated by measurement of CNQXresistant potentials or antidromic unit activities of pyramidal neurons. The estimated density of horizontal connections differed significantly between AC and VC. Therefore, it is very likely that the density of horizontal axon collaterals of pyramidal neurons is important in the amplification of polysynaptic and postsynaptic depolarization and of TS-induced LTP.

\section{Critical density of horizontal axon collaterals required for amplification of polysynaptic and postsynaptic depolarization and of TS-induced LTP}

Axon collaterals $>1 \mathrm{~mm}$ are well developed in VC (Gilbert, 1992). However, an effect of cutting of horizontal connections on TS-induced LTP was observed only in AC. Auditory pyramidal neurons tend to extend long-range axon collaterals through isofrequency bands (Reale et al., 1983; Ojima et al., 1991), which extend through the dorsoventral axis in rats (Sally and Kelly, 1988). However, the amplitude of TS-induced LTP in the coronal slices parallel to the isofrequency bands did not differ significantly from that in the horizontal slices cut orthogonally to the isofrequency bands. Therefore, long-range axon collaterals do not seem critical for TS-induced LTP. The slits, which caused a reduction in the amplitude of TS-induced LTP in AC, were $<500 \mu \mathrm{m}$ apart. Therefore, it is quite likely that axon collaterals located within $500 \mu \mathrm{m}$ from the soma determine the amplitude of TS-induced LTP in AC. This range might correspond to the area where accumulation of extracellular $\mathrm{K}^{+}$is elicited by TS, and neural excitability is facilitated (Leng et al., 1988). However, this hypothesis does not explain the differences in TS-induced LTP amplitude between AC and VC.

Alternatively, there might be a critical density of horizontal connections required for amplification of TS-induced LTP. Supragranular pyramidal neurons are connected to each other, and a single spike in the activity of one pyramidal neuron is sufficient to elicit measurable EPSPs in the target pyramidal neurons (Thomson et al., 1988; Mason et al., 1991). During TS, many pyramidal neurons are activated simultaneously, so that mutual recurrent connections may function as reverberating circuits. Such reverberating activities are observed in networks of cultured cortical neurons (Muramoto et al., 1993). Although long-lasting reverberating activity is unlikely to occur because of presynaptic depression and postsynaptic desensitization (Thomson and West, 1993), transient reverberation may be produced during TS if the gain of mutual recurrent connections between pyramidal neurons is larger than unity. The density of horizontal connections in AC is higher than that in $\mathrm{VC}$, and a high density of axon collaterals is expected to be present within a narrow area around the soma. TS-induced LTP seems to be amplified by local circuits located 
within a narrow area around the soma in AC. This coincidence between the high density of connections and amplification of TS-induced LTP is explained by assuming that the density of connections around the soma in $\mathrm{AC}$ is larger than the critical density at which the gain of mutual recurrent connections between pyramidal neurons is unity.

\section{Functional significance of local circuits specific to AC}

In the present study, we found well developed horizontal axon collaterals of pyramidal neurons in slices of AC. Because most of the local connections cut by the slicing are expected to be intact in vivo, characteristics of local circuits are expected to be clearer in vivo than in slices. The well developed horizontal connections in AC may have various functional implications. First, thalamocortical sensory inputs are amplified by recurrent intracortical circuits (Douglas et al., 1995), of which horizontal axon collaterals are main components. Well developed horizontal connections in $\mathrm{AC}$, therefore, facilitate postsynaptic depolarization induced by auditory inputs. Because postsynaptic depolarization is quite important in cortical plasticity (Frégnac et al., 1992; Shultz and Frégnac, 1992; Cruikshank and Weinberger, 1996), synaptic plasticity elicited by auditory inputs in vivo is probably facilitated by horizontal connections, as TS-induced LTP in slices is amplified. This characteristic feature of AC may explain marked synaptic plasticity elicited by learning in AC of adult animals (Ahissar et al., 1992; Edeline and Weinberger, 1993; Recanzone et al., 1993).

Intracortical connections between pyramidal neurons are expected to play an essential role in synchronized and oscillatory activities in the sensory cortex (Singer, 1993). Horizontal connections may mediate synchronization of neural activities in AC. The synchronized and oscillatory activities found in VC (Grey and Singer, 1989) have also been detected in AC by electrophysiological recording (Franowicz and Barth, 1995; Barth and MacDonald, 1996), optical recording (Fukunishi and Murai, 1995), and magnetic field recording (Tesche and Hari, 1993). Rhythmic activities may be generated in a single cortical neuron (Llinás et al., 1991). However, extensive synchronization between neurons requires the presence of well developed horizontal connections. A logical question derived from this consideration is whether many neurons connected with well developed horizontal axon collaterals in AC may simultaneously respond to auditory stimuli. As expected, scattered distribution of excited neurons during presentation of auditory stimuli can be observed in AC by optical recording (Fukunishi et al., 1992; Taniguchi et al., 1992). In contrast, high-resolution images of neural populations excited by visual stimuli in VC can be obtained through optical recording (Frostig et al., 1990; Bonhoeffer and Grinvald, 1991). These results suggest that neurons connected with well developed axon collaterals in AC might be suitable substrates for population coding of auditory information (Wang et al., 1995).

\section{REFERENCES}

Ahissar E, Vaadia E, Ahissar M, Bergman H, Arieli A, Abeles M (1992) Dependence of cortical plasticity on correlated activity of single neurons on behavioral context. Science 257:1412-1415.

Artola A, Singer W (1987) Long-term potentiation and NMDA receptors in rat visual cortex. Nature 330:649-652.

Artola A, Bröcher S, Singer W (1990) Different voltage-dependent thresholds for inducing long-term depression and long-term potentiation in slices of rat visual cortex. Nature 347:69-72.

Barth DS, MacDonald KD (1996) Thalamic modulation of highfrequency oscillating potentials in auditory cortex. Nature 383:78-81.

Blakemore C, Cooper GF (1970) Development of the brain depends on the visual environment, Nature 228:477-478.
Blomfield S (1974) Arithmetical operations performed by nerve cells. Brain Res 69:115-124.

Bonhoeffer T, Grinvald A (1991) Iso-orientation domains in cat visual cortex are arranged in pinwheel-like patterns. Nature 353:429-431.

Castro-Alamancos MA, Connors BW (1996) Short-term synaptic enhancement and long-term potentiation in neocortex. Proc Natl Acad Sci USA 93:1335-1339.

Castro-Alamancos MA, Donoghue JP, Connor BW (1995) Different forms of synaptic plasticity in somatosensory and motor areas of the neocortex. J Neurosci 15:5324-5333.

Chagnac-Amitai Y, Connors BW (1989) Horizontal spread of synchronized activity in neocortex and its control by GABA-mediated inhibition. J Neurophysiol 61:747-758.

Crair MC, Malenka RC (1995) A critical period for long-term potentiation at thalamocortical synapses. Nature 375:325-328.

Cruikshank SJ, Weinberger NM (1996) Receptive-field plasticity in the adult auditory cortex induced by Hebbian covariance. J Neurosci 16:861-875.

deCharms RC, Merzenich MM (1996) Primary cortical representation of sounds by the coordination of action-potential timing. Nature 381:610-613.

Douglas RJ, Koch C, Mahowald M, Martin KAC, Suarez HH (1995) Recurrent excitation in neocortical circuits. Science 269:981-985.

Edeline JM, Weinberger NM (1993) Receptive field plasticity in the auditory cortex during frequency discrimination training: selective retuning independent of task difficulty. Behav Neurosci 107:82-103.

Franowicz MN, Barth DS (1995) Comparison of evoked potentials and high-frequency (gamma-band) oscillating potentials in rat auditory cortex. J Neurophysiol 74:96-112.

Frégnac Y, Shultz D, Thorpe S, Bienenstock E (1992) Cellular analogs of visual cortical epigenesis. I. Plasticity of orientation selectivity. J Neurosci 12:1280-1300.

Frostig RD, Lieke EE, Ts'o DY, Grinvald A (1990) Cortical functional architecture and local coupling between neuronal activity and the microcirculation revealed by in vivo high-resolution optical imaging of intrinsic signals. Proc Natl Acad Sci USA 87:6082-6086.

Fukunishi K, Murai N (1995) Temporal coding in the guinea-pig auditory cortex as revealed by optical imaging and its pattern-time-series analysis. Biol Cybern 72:463-473.

Fukunishi K, Murai N, Uno H (1992) Dynamic characteristics of the auditory cortex of Guinea pigs observed with multichannel optical recording. Biol Cybern 67:501-509.

Gilbert CD (1992) Horizontal integration and cortical dynamics. Neuron 19:1-13.

Grey CM, Singer W (1989) Stimulus-specific neuronal oscillations in orientation columns of cat visual cortex. Proc Natl Acad Sci USA $86: 1698-1702$.

Hess G, Donoghue JP (1994) Long-term potentiation of horizontal connections provides a mechanism to reorganize cortical motor maps. J Neurophysiol 71:2543-2547.

Hirsch HVB, Spinelli DN (1970) Visual experience modifies distribution of horizontally and vertically oriented receptive fields in cats. Science 168:869-871.

Hirsch JA, Gilbert CD (1993) Long-term changes in synaptic strength along specific intrinsic pathways in the cat visual cortex. J Physiol (Lond) 461:247-262.

Hubel DH, Wiesel TN (1963) Receptive fields of cells in striate cortex of young, visually inexperienced kittens. J Neurophysiol 26:994-1002.

Iriki A, Pavlides C, Keller A, Asanuma H (1989) Long-term potentiation in the motor cortex. Science 245:1385-1387.

Kimura F, Nishigori A, Shirokawa T, Tsumoto T (1989) Long-term potentiation and $N$-methyl-D-aspartate receptors in the visual cortex of young rats. J Physiol (Lond) 414:125-144.

Kirkwood A, Lee H-K, Bear MF (1995) Co-regulation of long-term potentiation and experience-dependent synaptic plasticity in visual cortex by age and experience. Nature 375:328-331.

Krieg WJS (1964) Connections of the cerebral cortex. J Comp Neurol 84:221-323.

Kudoh M, Shibuki K (1994) Long-term potentiation in the auditory cortex of adult rats. Neurosci Lett 171:21-23.

Kudoh M, Shibuki K (1996a) Long-term potentiation of supragranular pyramidal outputs in the rat auditory cortex. Exp Brain Res 110:21-27.

Kudoh M, Shibuki K (1996b) LTP facilitated by extensive horizontal connections in the auditory cortex. Neurosci Res [Suppl] 20:S160.

Leng G, Shibuki K, Way SA (1988) Effects of raised extracellular potas- 
sium on the excitability of, and hormone release from, the isolated rat neurohypophysis. J Physiol (Lond) 99:591-605.

Llinás RR, Grace AA, Yarom Y (1991) In vitro neurons in mammalian cortical layer 4 exhibit intrinsic oscillatory activity in the $10-$ to $50-\mathrm{Hz}$ frequency range. Proc Natl Acad Sci USA 88:897-901.

Mason A, Nicoll A, Stratford K (1991) Synaptic transmission between individual pyramidal neurons of the rat visual cortex in vitro. J Neurosci 11:72-84.

Muramoto K, Ichikawa M, Kawahara M, Kobayashi K, Kuroda Y (1993) Frequency of synchronous oscillations of neural activity increases during development and is correlated to the number of synapses in cultured cortical neuron networks. Neurosci Lett 163:163-165.

Norwak L, Bregestovski P, Ascher P, Herbet A, Prochiantz A (1984) Magnesium gates glutamate-activated channels in mouse central neurons. Nature 307:462-465.

Ojima H, Honda CN, Jones EG (1991) Patterns of axon collateralization of identified supragranular pyramidal neurons in the cat auditory cortex. Cereb Cortex 1:80-94.

Reale RA, Brugge JF, Feng JZ (1983) Geometry and orientation of neural processes in cat primary auditory cortex (AI) related to characteristic-frequency maps. Proc Natl Acad Sci USA 80:5449-5453.

Recanzone GH, Schreiner CE, Merzenich MM (1993) Plasticity in the frequency representation of primary auditory cortex following discrimination training in adult owl monkeys. J Neurosci 13:87-103.

Sakai M, Kudoh M, Shibuki K (1995) A quick test for sound discrimination ability of rats in a single session after preparatory training. Neurosci Res 21:273-276.

Sakai M, Kudoh M, Shibuki K (1996) Fixation of enhanced sound discrimination ability in the rat. Neurosci Res [Suppl] 20:S238.

Sally SL, Kelly JB (1988) Organization of auditory cortex in the albino rat: Sound frequency. J Neurophysiol 59:1627-1638.

Shultz D, Frégnac Y (1992) Cellular analogs of visual cortical epigenesis. II. Plasticity of binocular integration. J Neurosci 12:1301-1318.
Singer W (1993) Synchronization of cortical activity and its putative role in information processing and learning. Annu Rev Physiol 55:349-374.

Taniguchi I, Horikawa J, Moriyama T, Nasu M (1992) Spatio-temporal pattern of frequency representation in the auditory cortex of guinea pigs. Neurosci Lett 146:37-40.

Tesche C, Hari R (1993) Independence of steady-state 40-Hz response and spontaneous $10-\mathrm{Hz}$ activity in the human auditory cortex. Brain Res 629:19-22.

Thomson AM, Deuchars J (1994) Temporal and spatial properties of local circuits in neocortex. Trends Neurosci 17:119-126.

Thomson AM, West DC (1993) Fluctuations in pyramid-pyramid excitatory postsynaptic potentials modified by presynaptic firing pattern and postsynaptic membrane potential using paired intracellular recordings in rat neocortex. Neuroscience 54:329-346.

Thomson AM, Girdlestone D, West DC (1988) Voltage-dependent currents prolong single axon postsynaptic potentials in layer III pyramidal neurons in rat neocortical slices. J Neurophysiol 60:1896-1907.

Toyama K, Matsunami K, Ohno T (1969) Antidromic identification of association, commissural and corticofugal efferent cells in cat visual cortex. Brain Res 14:513-517.

Wang X, Merzenich MM, Beitel R, Schreiner CE (1995) Representation of a species-specific vocalization in the primary auditory cortex of the common marmoset: temporal and spectral characteristics. J Neurophysiol 74:2685-2706.

Wiesel TN, Hubel DH (1963) Single-cell responses in striate cortex of kittens deprived of vision in one eye. J Neurophysiol 26:1003-1017.

Williams SM, Goldman-Rakic PS, Leranth C (1992) The synaptology of parvalbumin-immunoreactive neurons in the primate prefrontal cortex. J Comp Neurol 320:353-369.

Woolsey TA, Wann JR (1976) Areal changes in mouse cortical barrels following vibrissal damage at different postnatal ages. J Comp Neurol 170:53-66.

Zilles K (1985) The cortex of the rat. Berlin: Springer. 